\title{
Optimized codon usage enhances the expression and immunogenicity of DNA vaccine encoding Taenia solium oncosphere TSOL18 gene
}

\author{
YUAN-YUAN WANG ${ }^{1,2 *}$, XUE-LIAN CHANG ${ }^{1,2^{*}}$, ZHI-YONG TAO $^{1,2}$, XIAO-LI WANG ${ }^{1,2}$, YU-MENG JIAO ${ }^{1,2}$, \\ YONG CHEN $^{1,2}$, WEN-JUAN QI ${ }^{1,2}$, HUI XIA ${ }^{1,2}$, XIAO-DI YANG ${ }^{1,2}$, \\ XIN SUN $^{1,2}$, JI-LONG SHEN ${ }^{3}$ and QIANG FANG ${ }^{1,2}$ \\ ${ }^{1}$ Department of Microbiology and Parasitology; ${ }^{2}$ Anhui Key Laboratory of Infection and Immunity, \\ Bengbu Medical College, Bengbu, Anhui 233030; ${ }^{3}$ Department of Pathobiology, \\ Anhui Medical University, Hefei, Anhui 230032, P.R. China
}

Received March 20, 2014; Accepted January 21, 2015

DOI: $10.3892 / \mathrm{mmr} .2015 .3387$

\begin{abstract}
Cysticercosis due to larval cysts of Taenia solium, is a serious public health problem affecting humans in numerous regions worldwide. The oncospheral stage-specific TSOL18 antigen is a promising candidate for an anti-cysticercosis vaccine. It has been reported that the immunogenicity of the DNA vaccine may be enhanced through codon optimization of candidate genes. The aim of the present study was to further increase the efficacy of the cysticercosis DNA vaccine; therefore, a codon optimized recombinant expression plasmid pVAX1/TSOL18 was developed in order to enhance expression and immunogenicity of TSOL18. The gene encoding TSOL18 of Taenia solium was optimized, and the resulting opt-TSOL18 gene was amplified and expressed. The results of the present study showed that the codon-optimized TSOL18 gene was successfully expressed in CHO-K1 cells, and immunized mice vaccinated with opt-TSOL18 recombinant expression plasmids demonstrated opt-TSOL18 expression in muscle fibers, as determined by immunohistochemistry. In addition, the codon-optimized TSOL18 gene produced a significantly greater effect compared with that of TSOL18 and active spleen cells were markedly stimulated in vaccinated mice. ${ }^{3} \mathrm{H}$-thymidine incorporation was significantly greater in the opt-TSOL18 group compared with that of the TSOL18, pVAX and blank control groups $(\mathrm{P}<0.01)$. In conclusion, the eukaryotic expression vector containing the codon-optimized TSOL18 gene was
\end{abstract}

Correspondence to: Professor Qiang Fang, Department of Microbiology and Parasitology, Bengbu Medical College, 2600 Donghai Avenue, Bengbu, Anhui 233030, P.R. China E-mail: fq333@sohu.com

${ }^{*}$ Contributed equally

Key words: cysticercosis, TSOL18 gene, codon optimization, expression, immunogenicity successfully constructed and was confirmed to be expressed in vivo and in vitro. The expression and immunogenicity of the codon-optimized TSOL18 gene were markedly greater compared with that of the un-optimized gene. Therefore, these results may provide the basis for an optimized TSOL18 gene vaccine against cysticercosis.

\section{Introduction}

Cysticercosis, which is caused by the larva of Taenia solium, has affected humans in numerous developing regions worldwide $(1,2)$, and even in certain developed countries $(3,4)$. Cysticercosis is a serious public health problem as well as a great threat to the animal industry, which may result in tremendous economic damages. This disease has been identified by the World Health Organization as one to be eliminated worldwide (5). In the life-cycle of Taenia (T.) solium, swine serve as the intermediate host, while humans are intermediate as well as definitive hosts. Humans contract cysticercosis through ingesting raw or poorly cooked meat contaminated with $T$. solium larvae. These larvae mature to become tapeworms, which are parasites of the small intestine. The gravid proglottids of tapeworms, which are filled with eggs, are discharged with the feces of the hosts; under circumstances of poor hygiene, swine and humans may intake food and water polluted by eggs, which may lead to cysticercosis. Efforts, including drug therapy, environmental sanitation, enhanced management of feces and quarantine measures, have achieved only minimally positive results in disease control (6). Vaccination has been confirmed as a potentially valuable novel method for the prevention of $T$. solium transmission $(7,8)$. The TSOL18 gene, which encodes the T. solium-specific TSOL18 antigen, is a homologue of T. ovis To18 (9) and T. saginata TSA18 (10), and along with other oncosphere antigens, it was shown to contain a fibronectin type III domain (11). A previous study has shown that the TSOL18 antigen may be the most immunogenic and protective protein ever reported against cysticercosis (12-15). A number of different expression systems have previously been used to create proteins; however, 
species-specific variations in codon usage often serve as one of the primary influences of the results $(16,17)$. A study has demonstrated that highly expressed genes 'prefer' optimal codons (18) and the expression levels of optimized codons were markedly higher compared with those of non-optimal codons $(16,19,20)$. In order to increase immunogenicity of TSOL18 through higher recombinant protein production, it is necessary to optimize the codon in the expression host due to codon bias.

Therefore, the aim of the present study was to use codon optimization for the gene encoding TSOL18 of T. solium, construct an optimized plasmid vector (optimized pVAX1/TSOL18) for transfection and then evaluate the expression of the optimized gene in vivo and in vitro in order to determine its immunogenicity.

\section{Materials and methods}

Codon optimization, design and synthesis of the TSOL18 gene. According to the codon usage in mouse cells, 79 out of 130 amino acids of TSOL18 were modified into the most preferred ones, based on codon preference in host species, for mammalian using GeneOptimizer software (Geneart AG, Regensburg, Germany), without changing the amino acid sequence; then, the optimized full-length TSOL18 gene was synthesized by Jinsite Biotechnology Co. (Nanjing, China). The target fragments were amplified using polymerase chain reaction (PCR; S100 Thermal Cycler, Bio-Rad Laboratories, Inc., Hercules, CA, USA) and the following pair of $19 \mathrm{bp}$ primers: P1, 5'-ATGGTGTGCAGGTTCGCCC-3' and P2, 5'-GGATCCTCAGCTTCTCCTC-3', were constructed. Restriction endonucleases HindIII and BamHI (Fermentas, Vilnius, Lithuania) were added at the N-terminus and PCR was performed using the primers self-complemented through 30 cycles of pre-denaturizing $\left(95^{\circ} \mathrm{C}\right.$ for $\left.5 \mathrm{~min}\right)$, denaturizing $\left(94^{\circ} \mathrm{C}\right.$ for $\left.30 \mathrm{sec}\right)$, annealing $\left(55^{\circ} \mathrm{C}\right.$ for $\left.30 \mathrm{sec}\right)$ and extension $\left(72^{\circ} \mathrm{C}\right.$ for $\left.2 \mathrm{~min}\right)$. Reaction products were purified and preserved, then the optimized TSOL18 gene and expression vector (pVAX1) were double digested with HindIII and BamHI, respectively, and ligated with T4 DNA Ligase (Fermentas) to construct the optimized expression vector pVAX1/TSOL18. Following transfection into $E$. coli DH5 $\alpha$, the positive transfectants were selected, cultured on $5 \mathrm{ml}$ lysogeny broth medium (Oxoid Limited, Basingstoke, UK) (kanamycin $+10 \mu \mathrm{g} / \mathrm{ml}$ ) and confirmed through Hind $I I I / \mathrm{Bam} H I$ double digestion. The DNA sequence of the clones was confirmed by Shanghai Sheng Gong Biological Engineering Co., Ltd (Shanghai, China).

Chinese hamster ovary (CHO)-K1 cell culture and transfection. CHO-K1 cells (cat. no. CCL-61; American Type Culture Collection, Rockville, MD, USA) were cultured at $37^{\circ} \mathrm{C}$ in a humidified atmosphere with $5 \% \mathrm{CO}_{2}$ in $\mathrm{F} 12 \mathrm{~K}$ medium (Gibco-BRL, Carlsbad, CA, USA) with 10\% fetal calf serum (Gibco-BRL), $2 \mathrm{mM}$ l-glutamine and $1.5 \mathrm{~g} / 1 \mathrm{NaHCO}_{3}$. For the experiment $5 \times 10^{5}$ cells $/ \mathrm{ml}$ were cultured per plate and medium was replaced every $24 \mathrm{~h}$ prior to transfection. $\mathrm{CHO}-\mathrm{K} 1$ cells floating in F12K medium containing $10 \%$ fetal calf serum were seeded $\left(3.5 \times 10^{5}\right.$ cells/well) onto six-well cell culture plates and anchorage-dependent cells reached 50-70\% confluence.
Subsequently, $100 \mu \mathrm{l}$ Sofast ${ }^{\mathrm{TM}}$ lipofection transfection reagent (Sunma Biotechnology Co., Ltd., Xiamen, China) diluent was added to $100 \mu \mathrm{l}(1 \mu \mathrm{g} / \mathrm{ml})$ optimized plasmid pVAX1/TSOL18 and $100 \mu \mathrm{l}(1 \mu \mathrm{g} / \mathrm{ml})$ plasmid pVAX1 (Invitrogen Life Technologies, Carlsbad, CA, USA). The mixtures were then added to the wells of a CHO-K1 cell culture plate with mixing. Following transfection, plasmid-transfected $\mathrm{CHO}-\mathrm{K} 1$ cells were cultivated at $37^{\circ} \mathrm{C}$ in a humidified atmosphere with $5 \% \mathrm{CO}_{2}$ in $\mathrm{F} 12 \mathrm{~K}$ medium with $10 \%$ fetal calf serum for $48 \mathrm{~h}$, with replacement of the medium every $24 \mathrm{~h}$. Transfected cells were harvested and preserved at $-80^{\circ} \mathrm{C}$.

Reverse transcription (RT)-PCR analysis of optimized $p V A X 1 / \mathrm{TSOL} 18 \mathrm{mRNAs}$. Total RNA was extracted from CHO-K1 cells using TRIzol (Invitrogen Life Technologies) $48 \mathrm{~h}$ following transfection, according to the manufacturer's instructions. The RNA precipitate was pelleted through centrifugation at $11,500 \mathrm{x}$ g for $10 \mathrm{~min}$ and washed with $70 \%$ ethanol (Sigma-Aldrich, St. Louis, MO, USA). Following an additional centrifugation under identical conditions, the precipitate was dissolved in 20-30 $\mu 10.1 \%$ diethylpyrocarbonate (Sigma-Aldrich)-treated water and preserved at $-80^{\circ} \mathrm{C}$. cDNA was synthesized using Revert Aid $^{\mathrm{TM}} \mathrm{M}$-Mulv Reverse Transcriptase (Fermentas) with oligo (dT) primer $(1 \mu \mathrm{l})$ from the total RNA (4 mg) in a final volume of $20 \mu \mathrm{l}$, according to the manufacturer's instructions under the following conditions: $25^{\circ} \mathrm{C}$ for $5 \mathrm{~min}, 42^{\circ} \mathrm{C}$ for $60 \mathrm{~min}, 70^{\circ} \mathrm{C}$ for $5 \mathrm{~min}$ and then maintained at $4^{\circ} \mathrm{C}$. PCR was performed using $0.2 \mu \mathrm{l}$ cDNA obtained from the RT-reaction, described above, as a template. The primer used for the optimized TSOL18 gene was the same as above. The reaction mixture $(25 \mu \mathrm{l})$ contained $0.2 \mu \mathrm{g}$ cDNA template, $10 \mathrm{mM}$ deoxyribonucleotide, $0.5 \mu \mathrm{l}$ forward primer, $0.5 \mu \mathrm{l}$ reverse primer and $0.5 \mu \mathrm{l}$ Taq polymerase (PrimeSTAR HS PCR kit; Takara Bio, Inc., Tokyo, Japan). PCR was performed under the following conditions: Preheating at $95^{\circ} \mathrm{C}$ for $3 \mathrm{~min}$, denaturing at $94^{\circ} \mathrm{C}$ for $45 \mathrm{sec}$, annealing at $52^{\circ} \mathrm{C}$ for $45 \mathrm{sec}$ and extension at $72^{\circ} \mathrm{C}$ for 1 min. Following 30 cycles of amplification, the PCR products were analyzed using agarose gel electrophoresis.

Western blot analysis of optimized pVAX1/TSOL18 protein. Transfected cells $\left(1 \times 10^{7}\right)$ were added to $100 \mu 11 \mathrm{X}$ lysis buffer (1\% NP-40, $0.5 \%$ deoxycholate and $0.1 \%$ SDS; Sigma-Aldrich). Following boiling for $5 \mathrm{~min}$, centrifugation at $8,944 \mathrm{x} \mathrm{g}$ at $4^{\circ} \mathrm{C}$ for $5 \mathrm{~min}$ was performed. The whole suspension was then applied to $12 \%$ SDS-PAGE at $100 \mathrm{~V}$ for $120 \mathrm{~min}$, and the proteins were transferred to a nitrocellulose membrane (Millipore Corp., Bedford, MA, USA). Following blocking with 5\% skimmed milk (Oxoid Limited) in phosphate-buffered saline (PBS) at $4^{\circ} \mathrm{C}$ for $12 \mathrm{~h}$, the membranes were probed with 1:2,000 dilutions of the human anti-oncosphere polyclonal antibody at $37^{\circ} \mathrm{C}$ for $2 \mathrm{~h}$, followed by the addition of horseradish peroxidase (HRP)-conjugated goat anti-human polyclonal immunoglobulin G $(1: 1,000$; Wuhan Boster Biological Technology, Ltd., Wuhan, China) at $37^{\circ} \mathrm{C}$ for $2 \mathrm{~h}$. Reactive bands were visualized using diaminobenzidine (Wuhan Boster Biological Technology, Ltd.). Meanwhile, standard protein markers were cut off from the nitrocellulose membrane and dyed with Ponceaux (Amresco LLC, Solon, OH, USA) for 10-15 min. 
Immunohistochemical analysis. A total of 12 eight-week-old wild-type female BALB/c mice were purchased from Anhui Medical University (Hefei, China), and the experiment was approved by the Institutional Ethical Review Committee of Bengbu Medical College (Bengbu, China). The mice were fed with food and water ad libitum in a specific pathogen-free environment maintained at $23 \pm 1^{\circ} \mathrm{C}$, in a $12 \mathrm{~h}$ light/dark cycle. The mice were randomly assigned to four groups ( $\mathrm{n}=3 /$ group) and intramuscularly injected in the hind legs with $50 \mu$ Sofast $^{\mathrm{TM}}$ lipofection transfection reagent diluent. In order to improve the efficiency of gene transfer, bupivacaine $(80 \mu 1,6.7 \mathrm{mg} / \mathrm{ml}$; Sigma-Aldrich) was injected at the same sites to regenerate muscle $24 \mathrm{~h}$ prior to the injection of the plasmids. The groups were vaccinated as follows: Group A, empty control, $10 \mu \mathrm{l}$ $5 \%$ glucose (Sigma-Aldrich); group B, empty vector, $100 \mu \mathrm{g}$ pVAX1; group C, $100 \mu \mathrm{g}$ pVAX1/TSOL18; and group D, $100 \mu \mathrm{g}$ optimized pVAX1/TSOL18.

The 12 mice were sacrificed by $\mathrm{CO}_{2}$ inhalation $48 \mathrm{~h}$ following immunization to examine histological changes within the injection site. Injected muscle tissues were removed and fixed in $10 \%$ formalin (Sigma-Aldrich), embedded in paraffin (Polysciences, Inc., Warrington, PA, USA) and sectioned for microscopic examination. Muscle tissue sections were incubated at $4^{\circ} \mathrm{C}$ for $12 \mathrm{~h}$ with human anti-oncosphere polyclonal antibody at dilutions of 1:200, 1:300, 1:400 and $1: 500$, followed by the addition of alkaline phosphatase (ALP)-conjugated rabbit immunoglobulin G secondary antibody for immunohistochemistry. Binding of antibodies to the muscle sections was evaluated using light microscopy (BX41; Olympus Corporation, Tokyo, Japan) at magnifications of x100 and $x 400$. Slides were reviewed to evaluate the staining result of the protein by two pathologists who were blinded to the experiment at the First Affiliated Hospital of Bengbu Medical College (Anhui, China). Scores were determined by combining the proportion of positively stained muscle cells and the intensity of staining. Mice muscle cell proportions were scored as follows: 0 , no positive cells; $1,<10 \%$ positive cells; $2,10-35 \%$ positive cells; $3,35-75 \%$ positive cells; and $4,>75 \%$ positive cells. Staining intensity was graded on a four-point scale: 1 , no staining; 2, light yellow; 3, yellow brown; and 4, brown. Total scores of $\leq 1$ were regarded as negative (-), 2 as low expression $(+), 3$ as moderate expression $(++)$ and $\geq 4$ were taken as high expression $(+++)$.

Proliferation measurement by ${ }^{3} H$-thymidine. Eight-week-old wild-type female BALB/c mice were randomly assigned to four groups ( $\mathrm{n}=10 /$ group): Optimized pVAX1/TSOL18 (150 $\mu \mathrm{g} /$ injection), pVAX1/TSOL18 (150 $\mu \mathrm{g}$ /injection), pVAX1 empty vector (150 $\mu \mathrm{g}$ /injection) and PBS (150 $\mu \mathrm{g} /$ injection). The four groups were immunized intramuscularly in the hind legs for a total of three immunizations administered at two-week intervals. In order to improve the efficiency of gene transfer, $6.7 \mathrm{mg} / \mathrm{ml}$ bupivacaine was injected at the same sites to regenerate muscle $1 \mathrm{~h}$ prior to plasmid injection. This study was carried out in strict accordance with the recommendations in the Guide for the Care and Use of Laboratory Animals of the National Institutes of Health. The protocol was approved by the Committee on the Ethics of Animal Experiments of the Institute for Bengbu Medical College (permit no. LAEC-2009-027). All surgery was performed under sodium pentobarbital anesthesia, and all efforts were made to minimize suffering.

Two weeks following the final immunization, mice were sacrificed, spleens were excised and single viable cell suspensions were isolated. Erythrocytes were lysed by ammonium chloride (155 mM; Sigma-Aldrich) and splenocyte cultures from individual animals were prepared using a syringe plunger, pressing spleen tissue through a cell strainer. Following washing in RPMI 1640 media (Gibco-BRL), supplemented with $10 \%$ fetal bovine serum, $2 \mathrm{mmol} / 1$ glutamine, $1 \mathrm{mmol} / 1$ sodium pyruvate, $100 \mathrm{U} / \mathrm{ml}$ penicillin (Sigma-Aldrich), $100 \mu \mathrm{g} / \mathrm{ml}$ streptomycin (Sigma-Aldrich) and $12.5 \mathrm{U} / \mathrm{ml}$ nystatin (Sigma-Aldrich), lymphocytes were counted using trypan blue to identify viable cells and were resuspended to a concentration of $1 \times 10^{6}$ cells $/ \mathrm{ml}$. Cells $\left(2 \times 10^{5}\right.$ in $\left.200 \mu \mathrm{l}\right)$ were plated in 96-well plates and stimulated in triplicate using the corresponding TSOL18 protein $(1 \mu \mathrm{g} / \mu \mathrm{l}, 20 \mu \mathrm{l}$ per well; as previously prepared in our lab). The positive control was conA. Following $48 \mathrm{~h}$ of incubation at $37^{\circ} \mathrm{C}$ with $5 \% \mathrm{CO}_{2}$, cells were pulsed with $1 \mu \mathrm{Ci}{ }^{3} \mathrm{H}$-thymidine for $24 \mathrm{~h}$ to measure proliferation. Cells were then harvested and thymidine incorporation was determined using a liquid scintillation counter (FJ-2107P; Xi'an Nuclear Instrument Factory, Xi'an, China) at $72 \mathrm{~h}$.

Statistical analysis. Values are presented as the mean \pm standard error of the mean. All data were analyzed using SPSS 17.0 software (International Business Machines, Armonk, NY, USA). Comparisons between the means were made using a one-way analysis of variance. $\mathrm{P}<0.05$ was considered to indicate a statistically significant difference between values.

\section{Results}

Codon optimization, design and synthesis of the TSOL18 gene. A comparison of the TSOL18 gene and codon-optimized TSOL18 gene is shown in Fig. 1. A total of 79 out of 130 amino acids of TSOL18 were modified, and identical residues are marked in yellow.

Fig. 2 shows the results of the electrophoresis of optimized pVAX1/TSOL18 and recombinant plasmid-optimized pVAX1/TSOL18 following digestion with HindIII and BamHI. Following electrophoresis, two different sets of DNA fragments were detected. The band at $\sim 3,000 \mathrm{bp}$ indicated the full-length recombinant plasmid vector DNA and the band at $\sim 414 \mathrm{bp}$ indicated the optimized pVAX1/TSOL18 DNA. These results were consistent with the theoretical base-pair calculation and the fragments corresponding to the predicted DNA length. In addition, the inserted fragment (414 bp) was confirmed to be recombinant plasmid-optimized pVAX1/TSOL18, as determined using directed sequencing.

Expression of optimized TSOL18 in eukaryotic cells. The functions of optimized pVAX1/TSOL18 gene vaccine rely on its expression of a certain amount of protein. To verify whether the optimized pVAX1/TSOL18 gene could be cultured in the eukaryotic cell system, CHO-K1 cells were transfected and the mRNA expression of optimized TSOL18 was analyzed using RT-PCR. The results showed that the size of product was in accordance with the expected size ( 414 bp) (Fig. 3); the internal control $\beta$-actin was detected at similar 


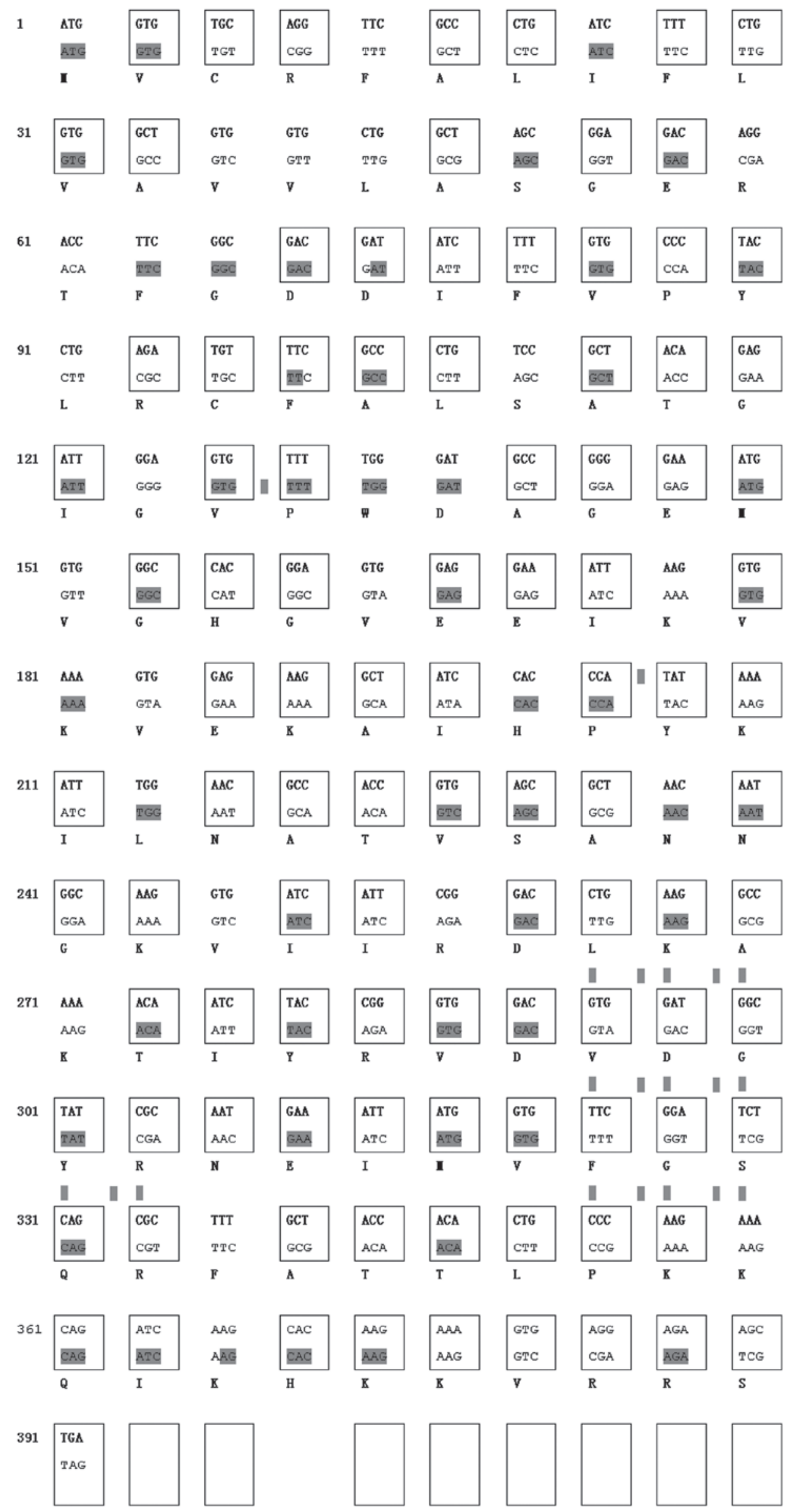

Figure 1. Alignment of TSOL18 wild-type nucleotide sequence with the optimized TSOL18 sequence. Identical residues are marked in grey. First line, TSOL18 nucleotide sequence; second line, codon optimized TSOL18 nucleotide sequence; and third line, amino acid sequence. 


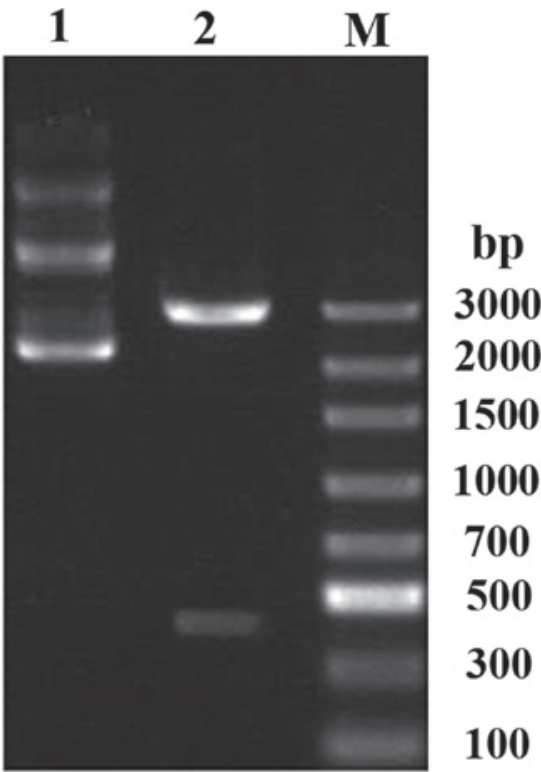

Figure 2. Protein expression of optimized pVAX1/TSOL18 and recombinant plasmid optimized pVAX1/TSOL18 following digestion with HindIII and BamHI. Lane 1, optimized pVAX1/TSOL18, two sets of DNA fragments were detected; lane 2, recombinant plasmid optimized pVAX1/TSOL18, the first band $(\sim 3,000 \mathrm{bp})$ indicated the full-length of recombinant plasmid vector DNA and the band ( 414 bp) indicated the optimized pVAX1/TSOL18 DNA; and lane 3, DNA molecular weight markers.

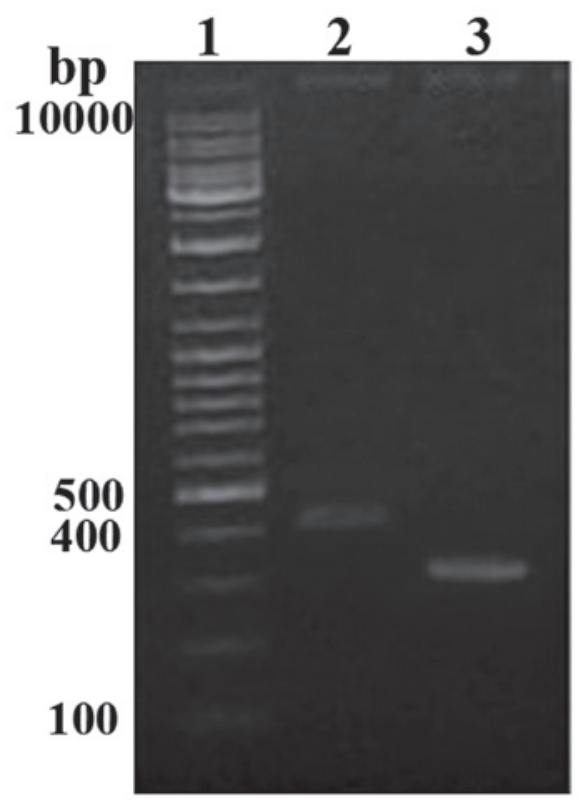

Figure 3. Expression of optimized pVAX1/TSOL18 messenger RNAs in eukaryotic cells detected using reverse transcription PCR. Agarose gel electrophoresis analysis showed: Lane 1, DNA marker; lane 2, PCR products of optimized pVAX1/TSOL18 gene ( 414 bp); and lane 3, PCR products of $\beta$-actin used as an internal control (300 bp). PCR, polymerase chain reaction.

levels ( $\sim 300 \mathrm{bp})$. These results demonstrated that the recombinant plasmid DNA vaccine expressed TSOL18 in eukaryotic cells. The cell lysate was then analyzed by western blotting, which revealed an obvious strong protein band at $18 \mathrm{kDa}$ in the optimized pVAX1/TSOL18 group, whereas the empty vector

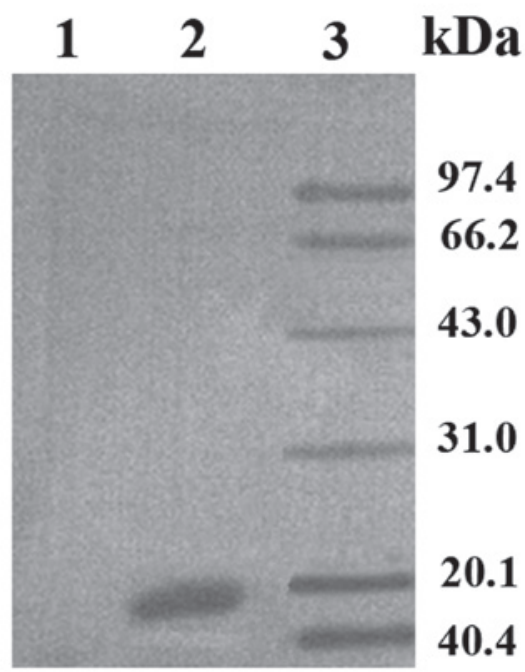

Figure 4. Expression products of the optimized pVAX1/TSOL18 protein analyzed by western blotting. Lane 1, pVAX1 group (no bands); lane 2, optimized pVAX1/TSOL18 group (an obvious strong protein band at $18 \mathrm{kDa}$ ); and lane 3, protein molecular weight markers.

pVAX1 group had no band (Fig. 4). These results demonstrated that the recombinant plasmid was able to express protein with immunocompetence in $\mathrm{CHO}-\mathrm{K} 1$ cells.

Expression of optimized TSOL18 in vivo. In order to determine whether optimized codon usage enhances TSOL18 gene expression in vivo, immunohistochemical analysis was performed to observe its expression in the hind leg muscles of mice (Fig. 5). The results revealed that immunostaining in the muscle of the optimized pVAX1/TSOL18 group was positive $(+++)$, with brown-yellow color pellets in the muscle fibers and more pellets were present in the optimized pVAX1/TSOL18 group compared with that of the pVAX1/TSOL18 group (++). In addition, the pVAX1 group muscle was negative for TSOL18 expression $(-\sim+)$.

Cell proliferation effect induced by the TSOL18 protein. In order to further verify whether the optimized pVAX1/TSOL18 induced a good immunity response, the cell proliferation rate was determined using ${ }^{3} \mathrm{H}$-thymidine. As shown in Fig. 6, ${ }^{3} \mathrm{H}$-thymidine incorporation was significantly greater in the optimized pVAX1/TSOL18 group compared with that in the pVAX1/TSOL18, pVAX1 and blank control groups $(\mathrm{P}<0.01)$. This suggested that the number of spleen cells in the optimized pVAX1/TSOL18 group stimulated by the TSOL18 protein was significantly increased compared with that in the pVAX1/TSOL18 group, indicating that the optimized pVAX1/TSOL18 demonstrated enhanced immunogenicity compared with that of pVAX1/TSOL18.

\section{Discussion}

Numerous factors may impact the expression of a protein, including the suitability of the promoter, codon bias, the position of the Shine-Dalgarno sequence and the stability of mRNA $(18,21)$. If a gene experiences strong selection pressure 

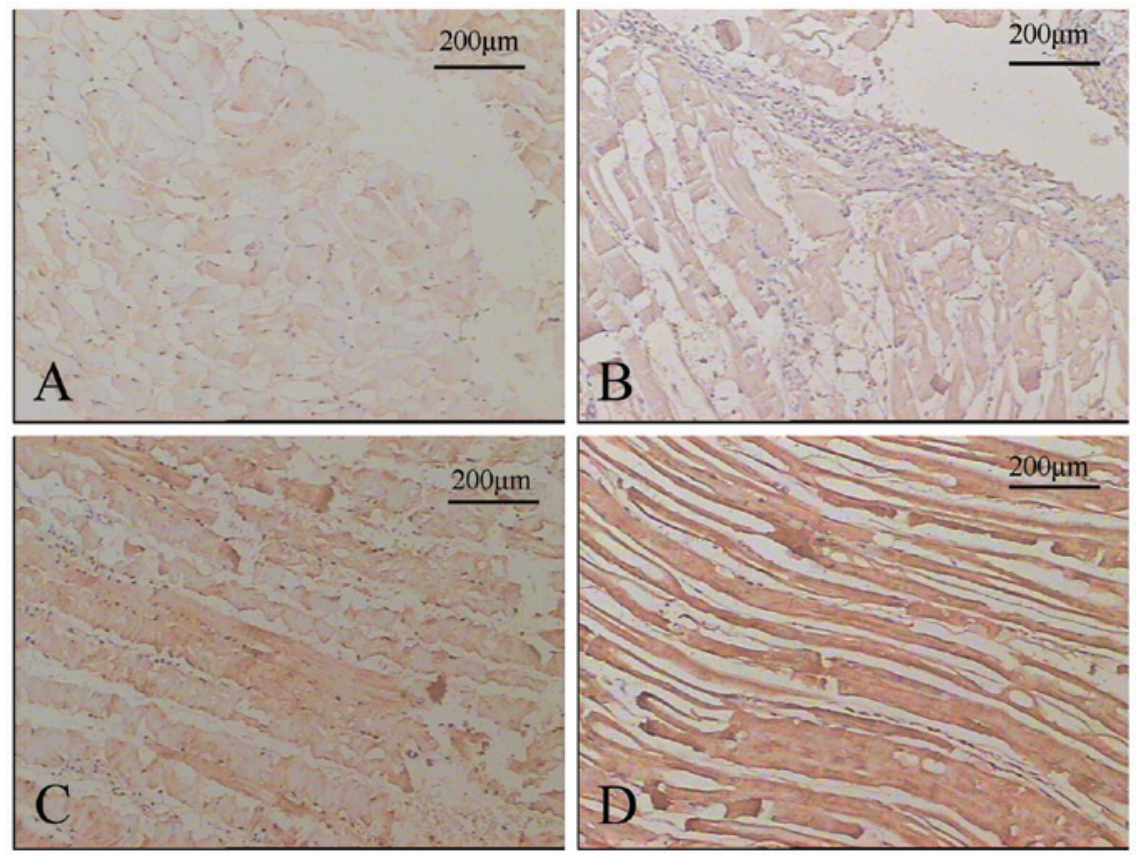

Figure 5. Immunohistochemical analysis of the expression of optimized TSOL18 in hind leg muscles of mice. (A) Negative control group injected with $10 \mu 1$ $5 \%$ glucose. No brown-yellow color pellets were observed in the muscle fibers (- +). (B) Control plasmid group injected with $100 \mu \mathrm{g}$ empty vector pVAX1 No brown-yellow color pellets were observed in the muscle fibers (- +). (C) pVAX1/TSOL18 group were injected with $100 \mu \mathrm{g}$ pVAX1/TSOL18. Moderate positive staining indicated by brown-yellow color pellets were observed in the muscle fibers (++). (D) Optimized pVAX1/TSOL18 group injected with $100 \mu \mathrm{g}$ optimized pVAX1/TSOL18. Strongly positive staining indicated by brown-yellow color pellets was observed in the muscle fibers (+++). Magnification, x100.

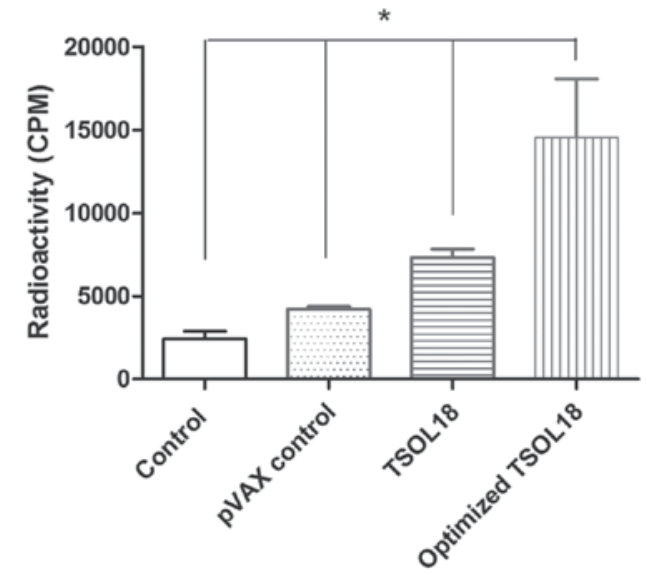

Figure 6. Cell proliferation was induced by the TSOL18 protein, as detected using ${ }^{3} \mathrm{H}$-thymidine. Values are presented as the mean \pm standard error of the mean. "P<0.01: TSOL18 group, $\mathrm{P}<0.01$ vs. blank control and PVAX groups; optimized TSOL18 group, $\mathrm{P}<0.01$ vs. blank control, PVAX and TSOL18 groups.

(such as high expression), it may have more usage codon bias and thus, a higher translation efficiency (22). Using codon modification, Zheng et al (23) successfully enhanced the expression of the glycoprotein gene in E. coli. In addition, Mani et al (24) reported that the codon adaptation index and guanine-cytosine content of the genes in optimized DNA was significantly enhanced.

In the present study, in order to improve TSOL18 expression, the codon optimization method was used to match codon frequencies in animal (mouse) organisms, modify ribosome binding sites and mRNA degradation sites, as well as adjust translational rates to allow various domains of the protein to fold properly (25). The codon usage for TSOL18 was mapped. RT-PCR amplification and gel electrophoresis revealed an obvious band at $\sim 414$ bp in the recombinant plasmid optimized pVAX1/TSOL18 transfected cells, indicating that the constructed plasmid had been successfully transfected into the $\mathrm{CHO}-\mathrm{K} 1$ cells. It has been reported that the induction of a specific immune response only occurs when the gene accessing the host body expresses a certain amount of protein $(26,27)$. Western blot analysis of the cell lysates revealed an obvious strong protein band at $18 \mathrm{kDa}$ in the optimized pVAX1/TSOL18 group, whereas the empty vector pVAX1 group had no band. These results demonstrated that the recombinant plasmid was able to express protein with immunocompetence in eukaryotic cells.

In the present study, pVAX1 was selected as the carrier vaccine expression vector, the safety of which has been approved by the Food and Drug Administration; in addition, CHO-K1 cells were previously found to be suitable for the expression of the pVAX1 plasmid (28). In the present study, the cationic polymer Sofast ${ }^{\mathrm{TM}}$ was applied in order to insert the reconstruction plasmid pVAX1/TSOL18 into CHO-K1 cells. Due to the high transfection efficiency and low cytotoxicity of Sofast ${ }^{\mathrm{TM}}$, the procedure is simple and quick (29).

DNA vaccines combine pathogen genes, encoding effective immunogens, and plasmids in order to get into the host cells through direct immunization and express the protective antigens (30). Thus, plasmid DNA vaccines must be injected into animal models in order to verify their effectiveness. In the present study, mice were selected instead of pigs as an animal model due to their small size, clear genetic background, easy use and exhibition of similar immune 
effects. At present, gene vaccine immunization is primarily performed using intramuscular injections, the gene gun method, mucosal immunization or intravenous and celiac injections (31). The route and delivery method used for genetic immunization studies have numerous implications affecting the outcome of the immune response. In the present study, intramuscular injection was selected as the method of immunization as experiments have shown that the DNA is readily absorbed by the host cells (Fig. 5D). Sheep were immunized intramuscularly with TO45w plasmid DNA in a study by Rothel et al (32), the results of which demonstrated high levels of immunoglobulin G1. In addition, BALB/c mice were immunized intramuscularly with plasmid DNA (pCDI-K45w, pcDI-K45 sec and pcDI-K45wTR) in a study by Drew et al (33), which reported that the cellular localization of the DNA vaccine antigen had a significant effect on the production of antibody.

The results of the present study revealed that immunostaining with anti-oncosphere antibodies in plasmid-injected muscle showed positive staining for TSOL18, whereas immunostaining in empty pVAX1-injected muscle showed negative results. In addition, an increased number of brown-yellow color pellets was observed in the optimized pVAX1/TSOL18 gene group compared with that of the pVAX1/TSOL18 group; this therefore indicated that the optimized pVAX1/TSOL18 gene expressed more protein compared with that of the un-optimized gene.

${ }^{3} \mathrm{H}$-thymidine, as a DNA precursor, can be used to indicate the degree of cell proliferation (34-36). In the present study, the lymphocyte transformation degree was determined according to ${ }^{3} \mathrm{H}$-thymidine radiation in the lymphocytes. This method is objective, sensitive and accurate (34-36). The results revealed that the number of spleen cells stimulated in the optimized pVAX1/TSOL18 gene group was significantly increased compared with that of the pVAX1/TSOL18 gene and empty contrast groups. These results indicated that following codon optimization, the quantity of protein expression increased and the active spleen cells were stimulated to rapidly proliferate, and therefore induced improved immunogenicity compared with that of the pVAX1/TSOL18 group.

In conclusion, the eukaryotic expression vector containing the codon-optimized TSOL18 gene was successfully constructed and expressed in vivo as well as in vitro. In addition, the expression and immunogenicity of the codon-optimized TSOL18 gene were markedly greater compared with those of the un-optimized gene in mice. These results provided the basis for developing an optimized TSOL18 gene vaccine against cysticercosis.

\section{Acknowledgements}

The authors would like thank Professor Junjie Sun, Department of Nuclear medicine, Bengbu Medical College and Professor Baiqing Li, MD, PhD, Department of Immunology, Bengbu Medical College, for their technical support. The present study was supported by grants from The Natural Science Foundation of China (no. 30600518), Anhui Provincial Natural Science Research Project (no. KJ2011B095) and Bengbu Medical College Fund (no. BY1001).

\section{References}

1. Shoji H, Hirai T, Shirakura T, et al: A case of cysticercosis with multiple lesions in the brain and femoral muscles. Kansenshogaku Zasshi 87: 608-612, 2013.

2. Rasamoelina-Andriamanivo H, Porphyre V and Jambou R: Control of cysticercosis in Madagascar: beware of the pitfalls. Trends Parasitol 29: 538-547, 2013.

3. Iacoangeli M, Moriconi E, Gladi M and Scerrati M: Isolated cysticercosis of the cauda equina. J Neurosci Rural Pract 4 (Suppl 1): S117-S119, 2013.

4. Sciutto E, Fragoso G, Fleury A, et al: Taenia solium disease in humans and pigs: an ancient parasitosis disease rooted in developing countries and emerging as a major health problem of global dimensions. Microbes Infect 2: 1875-1890, 2000.

5. Schantz PM, Cruz M, Sarti E and Pawlowski Z: Potential eradicability of taeniasis and cysticercosis. Bull Pan Am Health Organ 27: 397-403, 1993.

6. García HH, Gonzalez AE, Evans CA and Gilman RH; Cysticercosis Working Group in Peru: Taenia solium cysticercosis. Lancet 362: 547-556, 2003.

7. Drew DR, Lightowlers M and Strugnell RA: Vaccination with plasmid DNA expressing antigen from genomic or cDNA gene forms induces equivalent humoral immune responses. Vaccine 18: 692-702, 1999.

8. Solís CF, Ostoa-Saloma P, Lugo-Martínez VH, Johnston SA and Laclette JP: vaccination against murine cysticercosis by using a plasmid vector carrying Taenia solium paramyosin. Infect Immun 73: 1895-1897, 2005.

9. Harrison GB, Heath DD, Dempster RP, et al: Identification and cDNA cloning of two novel low molecular weight host-protective antigens from Taenia ovis oncospheres. Int J Parasitol 26: 195-204, 1996.

10. Lightowlers MW, Rolfe R and Gauci CG: Taenia saginata: vaccination against cysticercosis in cattle with recombinant oncosphere antigens. Exp Parasitol 84: 330-338, 1996.

11. Lightowlers MW, Flisser A, Gauci CG, Heath DD, Jensen O and Rolfe R: Vaccination against cysticercosis and hydatid disease. Parasitol Today 16: 191-196, 2000.

12. Rosas G, Fragoso G, Garate T, et al: Protective immunity against Taenia crassiceps murine cysticercosis induced by DNA vaccination with a Taenia saginata tegument antigen. Microbes Infect 4: 1417-1426, 2002.

13. Martinez-Ocaña J, Romero-Valdovinos M, de Kaminsky RG, Maravilla P and Flisser A: Immunolocalization of TSOL18 and TSOL 45-1A, the successful protective peptides against porcine cysticercosis, in Taenia solium oncospheres. Parasit Vectors 4: $3,2011$.

14. Flisser A, Gauci CG, Zoli A, et al: Induction of protection against porcine cysticercosis by vaccination with recombinant oncosphere antigens. Infect Immun 72: 5292-5297, 2004.

15. Cai X, Yuan G, Zheng Y, et al: Effective production and purification of the glycosylated TSOL18 antigen, which is protective against pig cysticercosis. Infect Immun 76: 767-770, 2008.

16. Zhou Z, Schnake P, Xiao L and Lal AA: Enhanced expression of a recombinant malaria candidate vaccine in Escherichia coli by codon optimization. Protein Expr Purif 34: 87-94, 2004.

17. Villalobos A, Ness JE, Gustafsson C, et al: Gene Designer: a synthetic biology tool for constructing artificial DNA segments. BMC Bioinformatics 7: 285, 2006.

18. Sharp PM and Devine KM: Codon usage and gene expression level in Dictyostelium discoideum: highly expressed genes do 'prefer' optimal codons. Nucleic Acids Res 17: 5029-5039, 1989.

19. Gao Z, Li Z, Zhang Y, et al: High-level expression of the Penicillium notatum glucose oxidase gene in Pichia pastoris using codon optimization. Biotechnol Lett 34: 507-514, 2012.

20. Lee MS, Hseu YC, Lai GH, et al: High yield expression in a recombinant $E$. coli of a codon optimized chicken anemia virus capsid protein VP1 useful for vaccine development. Microb Cell Fact 10: 56, 2011.

21. Gustafsson C, Govindarajan S and Minshull J: Codon bias and heterologous protein expression. Trends Biotechnol 22: 346-353, 2004.

22. Bulmer M: The selection-mutation-drift theory of synonymous codon usage. Genetics 129: 897-907, 1991.

23. Zheng JL, Jian GB and Guo XF: Codon modification for improvement in prokaryotic expression of glycoprotein gene in rabies virus. Chin J Zoonoses 26: 403-407, 2010. 
24. Mani I, Singh V, Chaudhary DK, Somvanshi P and Negi MP: Codon optimization of the major antigen encoding genes of diverse strains of influenza a virus. Interdiscip Sci 3: 36-42, 2011.

25. Raab D, Graf M, Notka F, Schödl T and Wagner R: The GeneOptimizer Algorithm: using a sliding window approach to cope with the vast sequence space in multiparameter DNA sequence optimization. Syst Synth Biol 4: 215-225, 2010.

26. Wang JX, Peng YC and Yao YX: Stage-specific expression of antigen encoded cDNA of cysticercus cellulosae. Chin J Parasitol Parasit Dis 2: 89-93, 1996.

27. Greene RM, Hancock K, Wilkins PP and Tsang VC: Taenia solium: molecular cloning and serologic evaluation of 14- and 18-kDa related, diagnostic antigens. J Parasitol 86: 1001-1007, 2000.

28. Hiszczyńska-Sawicka E, Li H, Xu JB, et al: Comparison of immune response in sheep immunized with DNA vaccine encoding Toxoplasma gondii GRA7 antigen in different adjuvant formulations. Exp Parasitol 124: 365-372, 2010.

29. Yang TC: Study on the transfection efficiency of the new cationic polymer transfection reagent: Sofast. Xia men da xue xue bao 43: 572-577, 2004.

30. Khan KH: DNA vaccines: roles against diseases. Germs 3 : 26-35, 2013.
31. Vibanco-perez N, Jimenez L, Mendoza-Hernandez G and Landa A: Characterization of a recombinant mu-class glutathione stransferase from Taenia solium. Parasitol Res 88: 394-404, 2002.

32. Rothel JS, Boyle DB, Both GW, et al: Sequential nucleic acid and recombinant adenovirus vaccination induces host-protective immune responses against Taenia ovis infection in sheep. Parasite Immunol 19: 221-227, 1997.

33. Drew DR, Lightowlers M and Strugnell RA: Humoral immune responses to DNA vaccines expressing secreted, membrane bound and non-secreted forms of the Tania ovis $45 \mathrm{~W}$ antigen. Vaccine 18: 2522-2532, 2000.

34. Dong XX, Zhang C, Yang XW, et al: Activities of treg cells stimulated by soluble adult worm antigen and egg antigen of Schistosoma japonicum. Zhongguo Xue Xi Chong Bing Fang Zhi Za Zhi 25: 146-150, 2013.

35. Hwang J, Yi M, Zhang X, Xu Y, Jung JH and Kim DK: Cytochalasin $\mathrm{B}$ induces apoptosis through the mitochondrial apoptotic pathway in HeLa human cervical carcinoma cells. Oncol Rep 30: 1929-1935, 2013.

36. Weng SX, Sui MH, Chen S, et al: Parthenolide inhibits proliferation of vascular smooth muscle cells through induction of G0/G1 phase cell cycle arrest. Zhejiang Univ Sci B 10: 528-535, 2009. 